\title{
Identifying current and remitted major depressive disorder with the Hurst exponent: a comparative study on two automated anatomical labeling atlases
}

\author{
Bin Jing ${ }^{1, *}$, Zhuqing Long ${ }^{1, *}$, Han Liu ${ }^{1, *}$, Huagang Yan ${ }^{1}$, Jianxin Dong ${ }^{1}$, Xiao Mo ${ }^{1}$, \\ Dan $\mathrm{Li}^{2}$, Chunhong Liu ${ }^{3,4}$ and Haiyun $\mathrm{Li}^{1}$ \\ ${ }^{1}$ School of Biomedical Engineering, Capital Medical University, Beijing, China \\ ${ }^{2}$ School of Software Engineering, Beijing University of Technology, Beijing, China \\ ${ }^{3}$ Acupuncture and Moxibustion Department, Beijing Hospital of Traditional Chinese Medicine Affiliated to Capital Medical \\ University, Beijing, China \\ ${ }^{4}$ Beijing Key Laboratory of Mental Disorders, Department of Radiology, Beijing Anding Hospital, Capital Medical University, \\ Beijing China \\ *These authors contributed equally to this work
}

Correspondence to: Haiyun Li, email: haiyunli@ccmu.edu.cn

Keywords: hurst exponent, automated anatomical labeling (AAL) atlas, resting-state fMRI, support vector machine, major depressive disorder

Received: January 07, $2017 \quad$ Accepted: July 17, $2017 \quad$ Published: August 03, 2017

Copyright: Jing et al. This is an open-access article distributed under the terms of the Creative Commons Attribution License 3.0 (CC BY 3.0), which permits unrestricted use, distribution, and reproduction in any medium, provided the original author and source are credited.

\section{ABSTRACT}

Major depressive disorder (MDD) is a leading world-wide psychiatric disorder with high recurrence rate, therefore, it is desirable to identify current MDD (CMDD) and remitted MDD (rMDD) for their appropriate therapeutic interventions. In the study, 19 cMDD, 19 rMDD and 19 well-matched healthy controls (HC) were enrolled and scanned with the resting-state functional magnetic resonance imaging (rs-fMRI). The Hurst exponent (HE) of rs-fMRI in AAL-90 and AAL-1024 atlases were calculated and compared between groups. Then, a radial basis function (RBF) based support vector machine was proposed to identify every pair of the CMDD, rMDD and HC groups using the abnormal HE features, and a leave-one-out cross-validation was used to evaluate the classification performance. Applying the proposed method with AAL1024 and AAL-90 atlas respectively, $87 \%$ and $84 \%$ subjects were correctly identified between CMDD and HC, $84 \%$ and $71 \%$ between rMDD and $H C$, and $89 \%$ and $74 \%$ between CMDD and rMDD. Our results indicated that the HE was an effective feature to distinguish CMDD and rMDD from HC, and the recognition performances with AAL1024 parcellation were better than that with the conventional AAL-90 parcellation.

\section{INTRODUCTION}

Major depressive disorder (MDD) is a common psychiatric disease, which is characterized by a low mood, worthlessness, anxiety and cognitive impairments $[1,2]$, and these symptoms are associated with the structural and functional impairments of some core brain regions [3]. By the year 2020, it will be the second leading global disease burden [4]. Besides, remitted MDD (rMDD) represents a not full recovery state of MDD, which also shows a tremendous effect on outcomes such as future relapse, morbidity and mortality [5]. Currently, no effective neurobiological markers or predictors are adopted to confirm the current MDD (cMDD) and rMDD in clinical practice, and the diagnosis of cMDD and rMDD are mainly based on the clinical symptoms and signs, and expert consensus. So it is desirable to develop effective brain imaging based diagnostic methods to identify cMDD and rMDD from healthy controls (HC), which could provide an objective perspective to recognize and understand the cMDD and rMDD. 
Magnetic resonance imaging (MRI) has attracted increasing attention for improving our understanding of the pathological mechanism and the underlying cognitive and affective dysfunctions in MDD [6]. A consistent finding is that MDD patients showed functional and structural abnormalities in limbic areas, prefrontal cortical regions and subcortical structures relative to $\mathrm{HC}$ [7-9], and the abnormalities in these brain regions may reflect the physiological characteristics of depressive patients. However, few studies have investigated the resting state brain abnormalities in rMDD patients. Yuan et al. reported that the remitted geriatric depression patients showed increased regional homogeneity ( $\mathrm{ReHo}$ ) in right median frontal gyrus, superior frontal gyrus and putamen, and decreased ReHo in parietal and temporal gyrus [10]. In our previous study, we found increased amplitude of lowfrequency fluctuation (ALFF)/fractional ALFF (fALFF) values in right putamen, and decreased ALFF/fALFF values in right precuneus and left lingual gyrus in remitted depression patients [11]. Though great progress has been made in cMDD and rMDD studies during the past decades, patient-specific diagnostic methods for cMDD and rMDD are still desperately needed.

In recent years, machine learning has been applied to identify MDD patients based on MRI signals. Fu et al. classified MDD patients from $\mathrm{HC}$ on the basis of their neural responses to the experiments of sad faces presentation, and accuracies of $74 \%$ and $76 \%$ were obtained with medium-intensity sad faces and highintensity sad faces respectively [1]. Hahn et al. used a Gaussian process classifier to identify MDD patients based on the integrating functional MRI (fMRI) data associated with affective and emotional processing, and acquired an accuracy of $83 \%$ [12]. Wei et al. adopted the Hurst exponent (HE) of twelve resting-state fMRI networks as the classification features, and achieved an accuracy of $90 \%[13,14]$. Marquand et al. investigated the functional neuroanatomy of verbal working memory as a potential diagnostic biomarker for depressive disorders, and got an accuracy rate of $68 \%$ [15]. Costafreda et al. used the support vector machine (SVM) algorithm on anatomical MRI data of MDD patients with pharmacological treatment, and yielded an accuracy of $89 \%$ [16]. To the best of our knowledge, few studies have utilized the machine learning method to discriminate every pair of the cMDD, rMDD and HC groups.

Appropriate brain parcellations have become a pursuing goal since the widespread applications of multi-modal MRI imaging [17-20], and different scaling atlases have been reported to result in considerable variations in relative studies [21,22]. Among the existing atlases, the Automated Anatomical Labeling (AAL-90) atlas is still the most popular atlas in the brain studies [23], and it has been widely used in the discriminative studies for different disorders [24-26]. Notably, some previous studies demonstrated that the structural-MRI characteristics based recognition rates were affected by the selection of brain atlases $[27,28]$. However, to the best of our knowledge, few studies have explored whether the fMRI characteristics (e.g. HE) based identification accuracies were also dependent on the atlas choice. In this study, AAL-1024 atlas was selected to compare with the conventional AAL-90 atlas for the following reasons. First, unlike other atlases, the AAL-1024 atlas is generated from the AAL-90 atlas, so it is easy to interpret and compare the abnormal regions between these two atlases. Second, the AAL-1024 atlas has 1024 subregions with identical size (approximate 40 voxels), so the influences of different sizes of subregions are avoidable. Moreover, considering some regions in the AAL-90 atlas are large, it is most likely that the signals from these regions are derived from several different functional subregions, which may influence the between-group findings, but the AAL-1024 atlas may better overcome this shortage.

Existing studies have demonstrated that spontaneous brain activities display scale-free dynamics, suggesting that the resting-state blood oxygen level-dependent (BOLD) signals show fractal-like properties [29]. HE, as an index ranging from 0 to 1 , could well display the scalefree dynamics via describing the self-similarity of a time series $[30,31]$. A HE bigger than 0.5 indicates a positively correlated or persistent behavior in the time series, and a HE smaller than 0.5 implies an anti-correlated time series, i.e., a decrease in time series will be generally followed by an increase in time series, while a HE equals to 0.5 indicating a random white-noise time series [32]. Recently, HE has been utilized widely to access pathological and physiological conditions. An increasing HE was reported to accompany with normal aging in bilateral hippocampus [33]. Besides, changes in HE were shown to be associated with autism spectrum disorders, cholinergic modulation and different personality traits [34-36]. However, the HE differences among cMDD patients, rMDD patients and $\mathrm{HC}$ groups still remain unknown, and it is also uncertain whether $\mathrm{HE}$ could be an effective feature to discriminate every pair of the cMDD patients, rMDD patients and HC groups.

In this paper, a radial basis function (RBF) based SVM method was proposed to identify every pair of cMDD, rMDD and HC groups using HE index. First, the HE characteristics of three groups were calculated by means of a rescaled range $(\mathrm{R} / \mathrm{S})$ analysis, and then the mean HE values in two AAL atlases (AAL-1024 and AAL-90) were computed and compared between groups. At last, the mean HE values of abnormal brain regions were served as classification features of the RBF based SVM algorithm to discriminate every pair of the three groups, and a leave-one-out cross-validation (LOOCV) was applied to evaluate the recognition performance. 


\section{RESULTS}

\section{Demographic and clinical information}

Table 1 summarized the demographic and clinical information about the cMDD patients, rMDD patients and HC groups. There were significant differences in Hamilton Depression Rating Scale (HAMD) scores between the cMDD and rMDD groups, but not in duration of illness and the number of episodes. No significant differences were observed in age and education level among three groups.

\section{Classification performance with two AAL atlases}

Applying the proposed method to the experimental MRI data with AAL-1024 atlas, $87 \%$ of the subjects were correctly classified between cMDD patients and $\mathrm{HC}$, together with an accuracy of $84 \%$ between rMDD patients and HC, and an accuracy of $89 \%$ between cMDD patients and rMDD patients. The detailed results were listed in Table 2. Taking every subject's predicted score as a threshold, the corresponding receiver operating characteristics (ROC) curves were acquired as shown in Figure 1, and the area under the ROC curves (AUCs) of the proposed method were $0.94,0.91$ and 0.92 , respectively. Table 3 listed the number of features retained in the proposed method per fold. Utilizing the proposed method to the experimental MRI data with AAL-90 atlas, $84 \%$ of the subjects were correctly classified between cMDD patients and $\mathrm{HC}$, together with an accuracy of $71 \%$ between rMDD patients and $\mathrm{HC}$, and an accuracy of $74 \%$ between cMDD patients and rMDD patients. Table 4 summarized the detailed results, and three ROC curves were also acquired as shown in Figure 2, and the AUCs of the proposed method were $0.85,0.64$ and 0.72 , respectively. The number of features retained in the proposed method per fold was showed in Table 5. Besides, all $P$ values of the permutation test were less than 0.01 , indicating the classification accuracies were reliable, and the relevant statistical results were showed in Figure 3 and Figure 4

\section{Between-group differences in $\mathrm{HE}$}

The most discriminative brain regions for group separation with AAL-1024 atlas and AAL-90 atlas were showed in Figure 5. When classifying cMDD patients from $\mathrm{HC}$, the most informative regions mainly contained left insula, bilateral cingulate gyrus, left middle temporal gyrus, left superior temporal gyrus, bilateral supplementary motor area and right superior parietal cortex. When classifying rMDD patients from $\mathrm{HC}$, the brain regions with great discriminative power mostly included bilateral insula, right cingulate gyrus, left superior frontal gyrus and left superior temporal gyrus.
While applying the proposed method to discriminate between cMDD patients and rMDD patients, the most informative brain regions were predominantly located in left middle frontal gyrus, bilateral middle occipital gyrus, right superior parietal cortex, and right inferior parietal lobule.

\section{DISCUSSION}

In this paper, a RBF based SVM algorithm was proposed to discriminate every pair of the cMDD patients, rMDD patients and $\mathrm{HC}$ groups using the $\mathrm{HE}$ of resting-state fMRI. Compared with the AAL-90 atlas, the proposed method with AAL-1024 atlas obtained better recognition performances, and yielded an accuracy of $87 \%$ between cMDD patients and HC, $84 \%$ accuracy between rMDD patients and $\mathrm{HC}$ and $89 \%$ accuracy between cMDD patients and rMDD patients.

The proposed classification method has several advantages. First, the RBF kernel function is simple (less parameters), and it has few numerical problems $\left(0<K\left(X, X_{i}\right)<1\right)$. Unlike linear kernel function, it can deal with the case when the relationship between features and labels is nonlinear [37]. Second, a grid search algorithm, which has a high learning accuracy and can be implemented with parallel processing, was utilized to optimize the two parameters of SVM, and it significantly improved the classification performance. Third, two-sample two-tailed $t$ tests were used to select out the discriminative features, which also has an important impact on the final performance. Several existing studies have demonstrated that correctly reducing the number of features can not only improve the classification performance but also speed up the computation $[38,39]$. Meanwhile, considering the feature selection process was only constrained on the training set of each LOOCV fold, it could reduce overfitting of the classifier [24]. Besides, the total 90 and $1024 \mathrm{HE}$ classification features were also tested using our proposed method respectively, and the classification accuracies without feature selection between any two groups were lower than $70 \%$, which were notably lower than that with feature selection. In addition, the split-half validation, which divided every pair of cMDD, rMDD and $\mathrm{HC}$ into two groups, one group for training ( 9 subjects in each category) and one group for testing (10 subjects in each category), and the model generated by the training set were used to classify the testing set. With AAL-90 and AAL-1024 atlas respectively, we obtained accuracies of $70 \%$ and $80 \%$ between cMDD and $\mathrm{HCs}, 80 \%$ and $90 \%$ between rMDD and $\mathrm{HCs}, 70 \%$ and $85 \%$ between cMDD and rMDD. At last, considering that using a well-diagnosed but heterogeneous group of patients with different severity levels of clinical symptoms while not excluding medicated patients could offer a more realistic estimation of the proposed classification method for MDD [12], therefore, we combined the cMDD and 
Table 1: Participant demographic and clinical characteristics

\begin{tabular}{lcccc}
\hline Variables (Mean \pm SD) & cMDD & rMDD & HC & P Values \\
\hline Number of subjects & 19 & 19 & 19 & - \\
Age (years) & $34.84 \pm 13.58$ & $37.58 \pm 12.69$ & $36.84 \pm 12.69$ & $0.79^{\mathrm{a}}$ \\
Education (years) & $12.84 \pm 3.18$ & $12.68 \pm 2.93$ & $13.74 \pm 2.16$ & $0.46^{\mathrm{a}}$ \\
Duration of illness (years) & $6.90 \pm 8.34$ & $7.37 \pm 5.53$ & & $0.84^{\mathrm{b}}$ \\
HAMD & $21.65 \pm 4.50$ & $4.63 \pm 2.57$ & & $0.00^{\mathrm{b}}$ \\
Number of depressive episodes & $2.68 \pm 1.95$ & $2.39 \pm 1.80$ & & $0.64^{\mathrm{b}}$ \\
Antidepressants & 23 & 29 & \\
Mood-stabilizer & 1 & 1 & \\
Antipsychotics & 4 & 6 & & \\
Benzodiazepines & 2 & 1 & & \\
Medication-free & 3 & 1 & & \\
\hline
\end{tabular}

SD: standard deviation; cMDD: current major depressive disorder; rMDD: remitted major depressive disorder; HC: healthy controls; HAMD: Hamilton Depression Rating Scale.

${ }^{a}$ The $P$ values were obtained by one-way ANOVA.

${ }^{\mathrm{b}}$ The $P$ values were obtained by two-sample two-tailed $t$-tests.

Table 2: The classification performance in every pair of cMDD, rMDD and HC with AAL-1024 atlas

\begin{tabular}{lccc}
\hline Groups & Accuracy & Sensitivity & Specificity \\
\hline cMDD vs. HC & $87 \%$ & $84 \%$ & $89 \%$ \\
rMDD vs. HC & $84 \%$ & $89 \%$ & $79 \%$ \\
cMDD vs. rMDD & $89 \%$ & $84 \%$ & $95 \%$ \\
\hline
\end{tabular}

rMDD patients as one group, and then discriminated the new combined group from HC. An accuracy of $75 \%$ was obtained using AAL-90 atlas, and $81 \%$ accuracy was achieved utilizing the AAL-1024 atlas, which reflected that our HE-based recognition method could effectively discriminate most MDD patients with heterogeneous symptoms and medication.
HE has become a widely used complexity index in recent resting-state fMRI studies. For example, it had been used as the diagnostic biomarkers for Alzheimer's disease [30], Autism Spectrum Disorders and Schizophrenia [34, 40]. These studies implied the possibility of $\mathrm{HE}$ as an independent discriminative feature in other psychiatric diseases. In a previous study, the HE

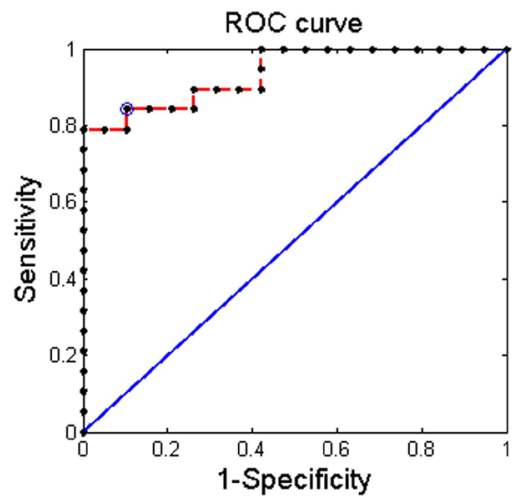

A

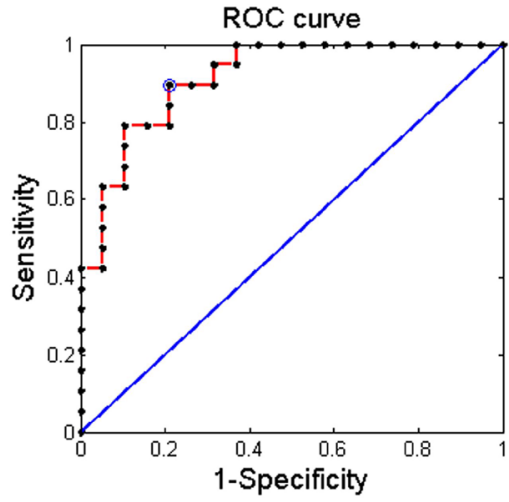

B

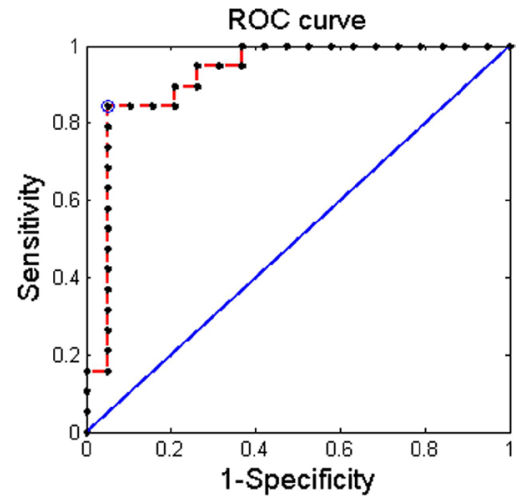

C

Figure 1: The receiver operating characteristics (ROC) curves of the proposed method with AAL-1024 atlas. (A) Between cMDD patients and HC; (B) Between rMDD patients and HC; (C) Between rMDD patients and cMDD patients. 
Table 3: The number of features retained in the proposed method per fold with AAL-1024 atlas

\begin{tabular}{|c|c|c|c|}
\hline Fold & Between cMDD and HC & Between rMDD and HC & Between cMDD and rMDD \\
\hline 1 & 75 & 73 & 63 \\
\hline 2 & 72 & 84 & 73 \\
\hline 3 & 78 & 76 & 70 \\
\hline 4 & 82 & 84 & 68 \\
\hline 5 & 73 & 71 & 74 \\
\hline 6 & 71 & 64 & 65 \\
\hline 7 & 86 & 89 & 71 \\
\hline 8 & 72 & 64 & 63 \\
\hline 8 & 75 & 70 & 66 \\
\hline 10 & 67 & 70 & 64 \\
\hline 11 & 71 & 69 & 69 \\
\hline 12 & 66 & 86 & 66 \\
\hline 13 & 71 & 71 & 59 \\
\hline 14 & 76 & 78 & 61 \\
\hline 15 & 76 & 64 & 61 \\
\hline 16 & 74 & 71 & 65 \\
\hline 17 & 69 & 68 & 57 \\
\hline 18 & 74 & 73 & 67 \\
\hline 19 & 74 & 81 & 62 \\
\hline 20 & 82 & 78 & 66 \\
\hline 21 & 79 & 79 & 70 \\
\hline 22 & 77 & 82 & 68 \\
\hline 23 & 78 & 66 & 62 \\
\hline 24 & 73 & 76 & 58 \\
\hline 25 & 69 & 73 & 67 \\
\hline 26 & 92 & 78 & 66 \\
\hline 27 & 73 & 67 & 69 \\
\hline 28 & 65 & 82 & 65 \\
\hline 29 & 69 & 80 & 57 \\
\hline 30 & 76 & 82 & 58 \\
\hline 31 & 80 & 69 & 69 \\
\hline 32 & 73 & 85 & 73 \\
\hline 33 & 87 & 62 & 63 \\
\hline 34 & 62 & 74 & 58 \\
\hline 35 & 71 & 75 & 63 \\
\hline 36 & 73 & 68 & 65 \\
\hline 37 & 86 & 67 & 63 \\
\hline 38 & 83 & 66 & 61 \\
\hline
\end{tabular}

was used to recognize the MDD patients from HC [13], and the mean $\mathrm{HE}$ values of twelve independent networks were selected as the discriminative features, but some networks (e.g. auditory, lateral visual network) that may be unrelated to MDD symptoms showed large weights among all features in the discrimination, which made the recognition rate seem not sound enough. By using the AAL-1024 atlas and AAL-90 atlas, our proposed method could find the more precise between-group features than the independent networks, and the reported regions may be more closely correlated with the MDD. Additionally, the physiological significance of HE is still uncertain, and 


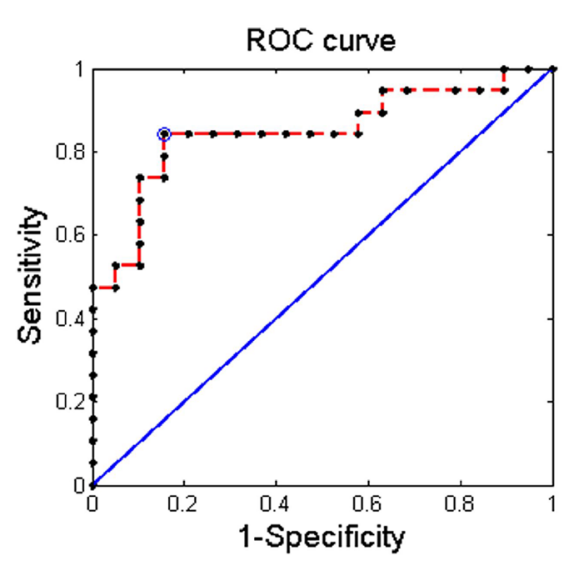

A

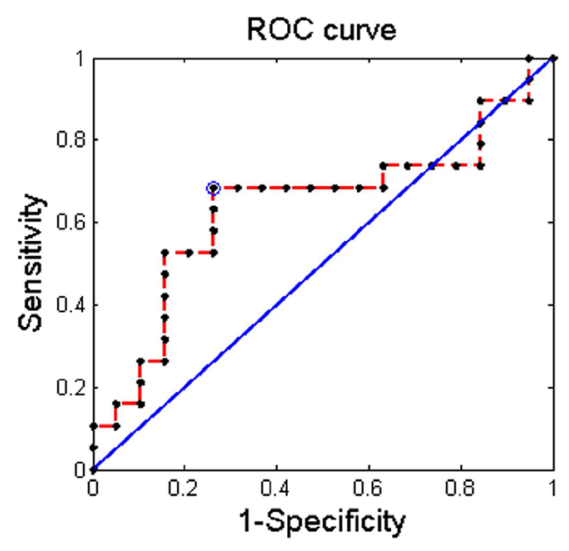

B

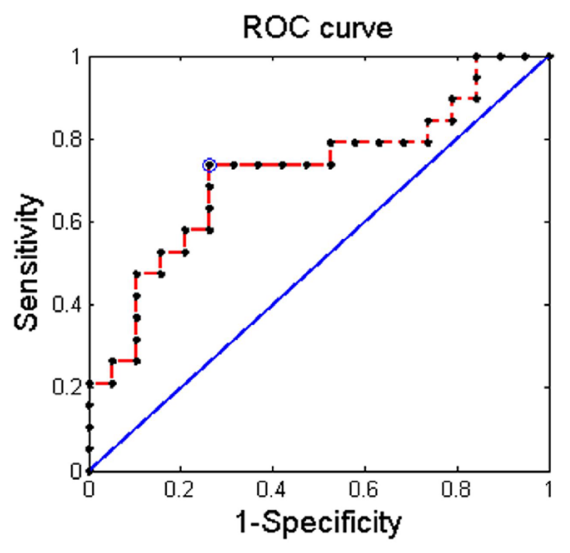

C

Figure 2: The receiver operating characteristics (ROC) curves of the proposed method with AAL-90 atlas. (A) Between cMDD patients and HC; (B) Between rMDD patients and HC; (C) Between rMDD patients and cMDD patients.

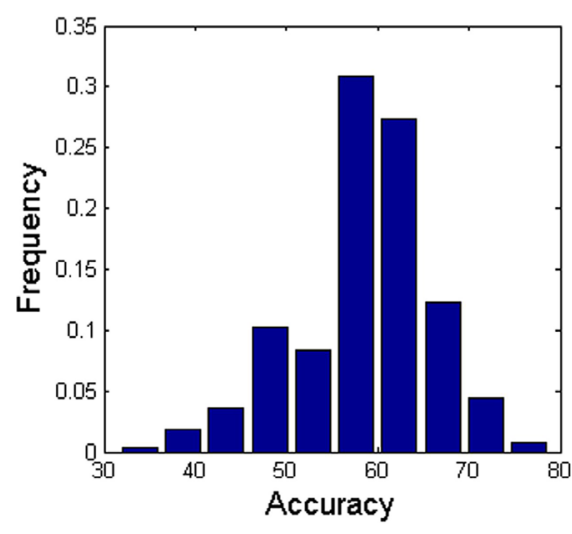

A

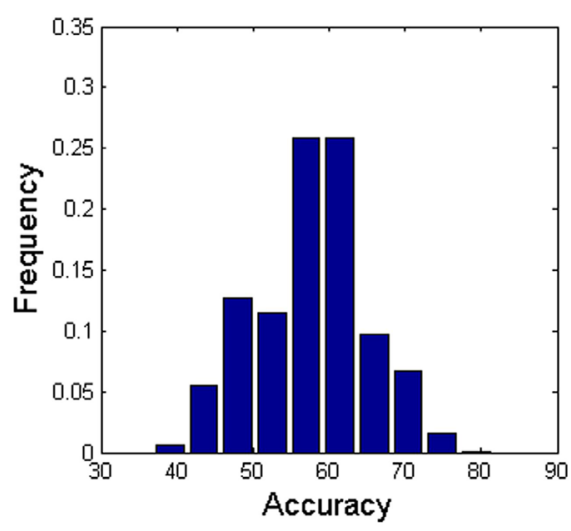

B

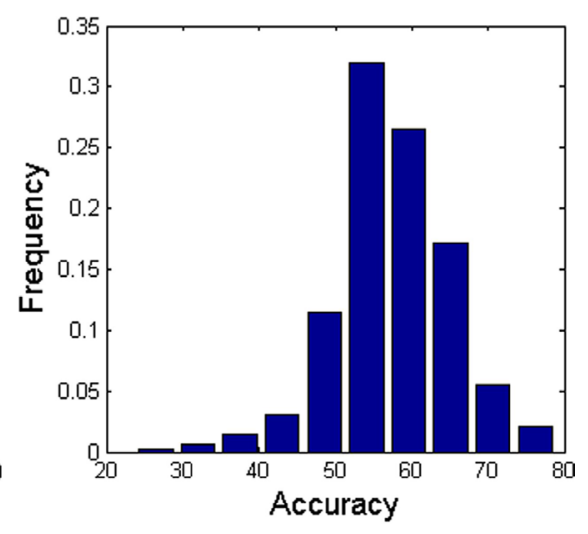

C

Figure 3: The permutation distributions of accuracies with AAL-1024 atlas. (A) Between cMDD patients and HC; (B) Between rMDD patients and HC; (C) Between rMDD patients and cMDD patients.

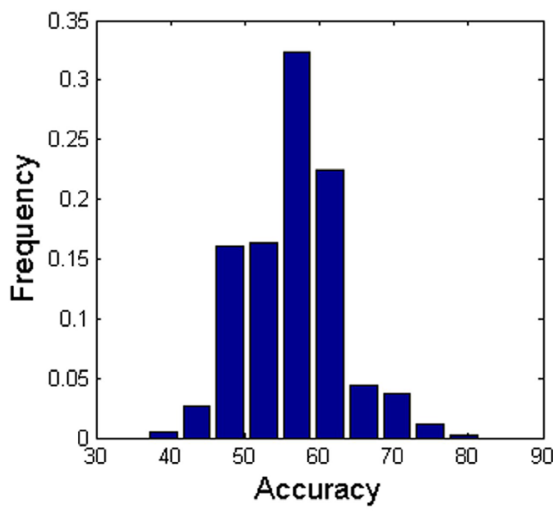

A

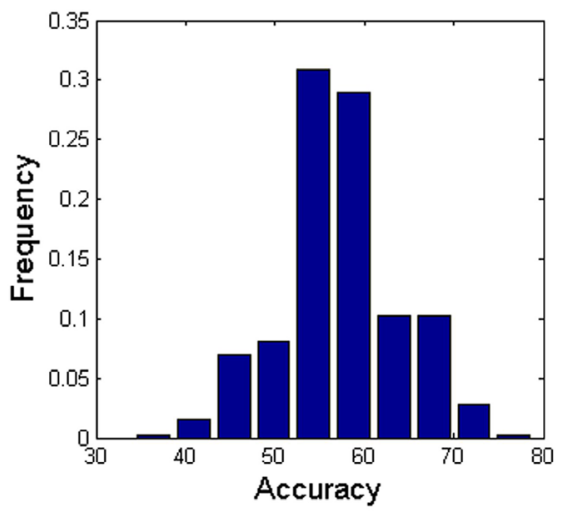

B

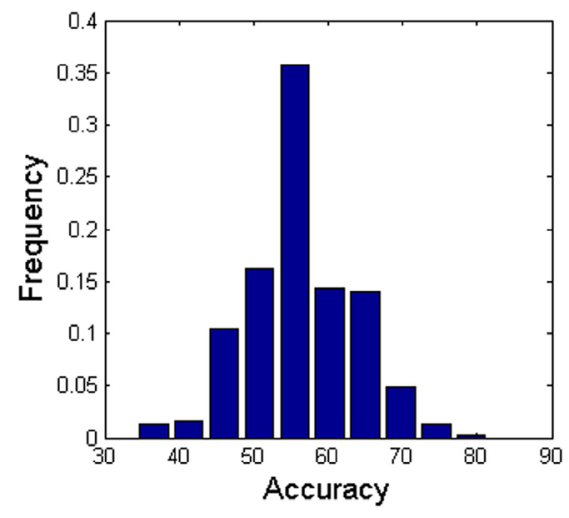

C

Figure 4: The permutation distributions of accuracies with AAL-90 atlas. (A) Between cMDD patients and HC; (B) Between rMDD patients and HC; (C) Between rMDD patients and cMDD patients. 
Table 4: The classification performance in every pair of cMDD, rMDD and HC with AAL-90 atlas

\begin{tabular}{lccc}
\hline Groups & Accuracy & Sensitivity & Specificity \\
\hline cMDD vs. HC & $84 \%$ & $84 \%$ & $84 \%$ \\
rMDD vs. HC & $71 \%$ & $68 \%$ & $74 \%$ \\
cMDD vs. rMDD & $74 \%$ & $74 \%$ & $74 \%$ \\
\hline
\end{tabular}

Table 5: The number of features retained in the proposed method per fold with AAL-90 atlas

\begin{tabular}{|c|c|c|c|}
\hline Fold & Between cMDD and HC & Between rMDD and HC & Between cMDD and rMDD \\
\hline 1 & 8 & 5 & 3 \\
\hline 2 & 9 & 7 & 3 \\
\hline 3 & 9 & 5 & 5 \\
\hline 4 & 7 & 6 & 5 \\
\hline 5 & 7 & 7 & 4 \\
\hline 6 & 8 & 3 & 4 \\
\hline 7 & 11 & 8 & 6 \\
\hline 8 & 8 & 4 & 4 \\
\hline 8 & 8 & 5 & 6 \\
\hline 10 & 8 & 3 & 4 \\
\hline 11 & 8 & 5 & 7 \\
\hline 12 & 9 & 7 & 5 \\
\hline 13 & 8 & 5 & 3 \\
\hline 14 & 10 & 7 & 5 \\
\hline 15 & 8 & 4 & 3 \\
\hline 16 & 8 & 4 & 5 \\
\hline 17 & 10 & 7 & 3 \\
\hline 18 & 7 & 4 & 4 \\
\hline 19 & 7 & 6 & 6 \\
\hline 20 & 9 & 5 & 6 \\
\hline 21 & 9 & 9 & 4 \\
\hline 22 & 8 & 5 & 4 \\
\hline 23 & 8 & 4 & 3 \\
\hline 24 & 9 & 5 & 3 \\
\hline 25 & 8 & 3 & 4 \\
\hline 26 & 7 & 3 & 4 \\
\hline 27 & 10 & 7 & 5 \\
\hline 28 & 8 & 9 & 4 \\
\hline 29 & 8 & 6 & 4 \\
\hline 30 & 9 & 6 & 3 \\
\hline 31 & 9 & 6 & 4 \\
\hline 32 & 8 & 8 & 8 \\
\hline 33 & 8 & 3 & 6 \\
\hline 34 & 7 & 4 & 3 \\
\hline
\end{tabular}


$36-8$

it is hard to interpret the between-group abnormalities definitely. Future studies should be conducted to confirm the physiological significance of HE through the multimodal imaging validations in the animal model.

In this paper, we separately discriminated every pair of cMDD patients, rMDD patients and HC groups with two AAL atlases, and found that the classification performance with AAL-1024 atlas was significantly better than that with AAL-90 atlas, which may arise from the differences in the discriminative features. To clearly elucidate the differences of the discriminative features between AAL1024 and AAL-90 atlas, three tables were added in the Supplementary Tables 1, 2, and 3 to list all the stable discriminative features in LOOCV for the between-group classifications. From the tables, two advantages could be found with AAL-1024 atlas: First, all abnormal regions reported by AAL- 90 were also detected by AAL-1024, and the discriminative features could be further located into more precise position by AAL-1024 atlas. For example, AAL-1024 atlas could discover more than one abnormal subregion in the regions reported by AAL-90, and this may be attributed to the existence of functional heterogeneity in AAL-90 parcellation, especially for some large regions.
Second, AAL-1024 atlas could also detect some abnormal regions overlooked by AAL-90 atlas, therefore, more discriminative features were obtained by AAL-1024 than AAL-90. Moreover, these new features discovered by AAL-1024 mainly lied in the default mode network and the limbic system, which highly correlated with the pathology of major depressive disorder [11, 41, 42]. In addition, to further display the advantages of AAL1024 atlas, the Fisher score method [43] was applied to all candidate features to detect whether the features revealed by AAL-1024 atlas could provide more discriminative power over the AAL-90 atlas. To have a comprehensive and direct comparison, the rearranged 90 features from AAL-90 atlas and the prior 90 features from AAL-1024 atlas were compared in Fisher score. From Figure 1 and Supplementary Figure 1 in Supplementary Material, the Fisher score of AAL-1024 was found significant larger than that of AAL-90, which again indicated the advantage of AAL-1024 atlas over AAL-90 atlas. Hurst exponent is a functional index for resting state fMRI, but the widely used AAL-90 atlas is generated based on anatomical information, therefore, AAL-90 atlas may be not sensitive to the functional abnormalities in local regions. Although
$\mathbf{L}$
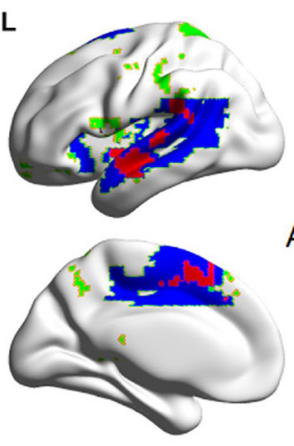

A
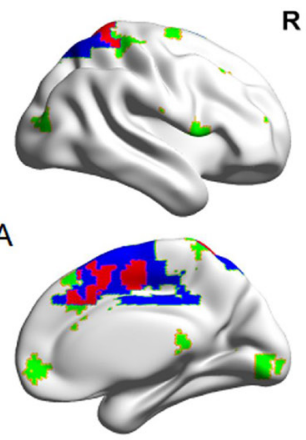

L
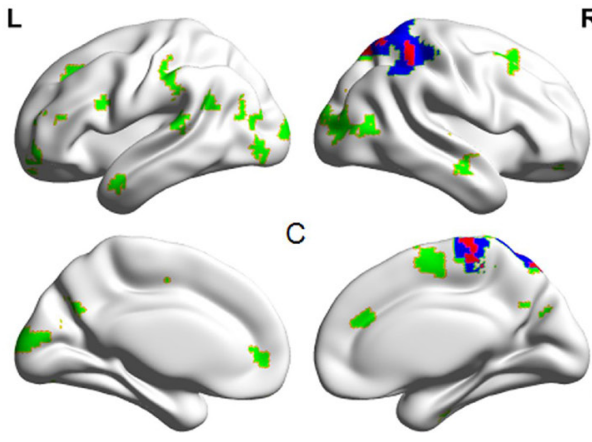

C

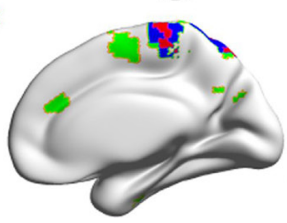

$\mathbf{R}$
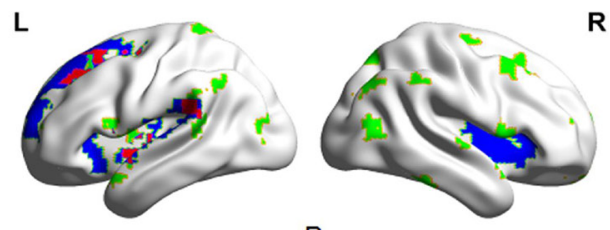

B
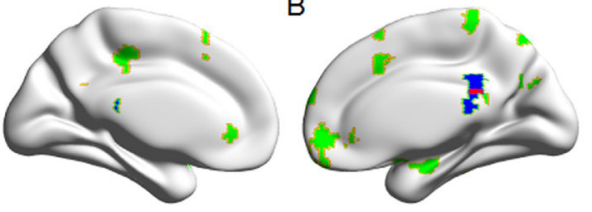

$\mathbf{R}$

$\mathbf{R}$

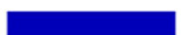

discriminative features with AAL-90

discriminative features with AAL-1024

intersection of discriminative features

with AAL 90/1024

Figure 5: The HE differences in every pair of cMDD patients and rMDD patients and HC. (A) Between cMDD patients and $\mathrm{HC}$; (B) Between rMDD patients and HC; (C) Between rMDD patients and cMDD patients. 
AAL-1024 atlas is also not a functional brain atlas, it partitions the whole brain into 1024 subdivisions with relative small size, therefore, it could largely improve the sensitivity to the local functional anomaly but also incorporate the local anatomical information.

In this study, the abnormal regions detected by the HE analysis in CMDD and rMDD patients turned out to be informative. Compared with HC, both cMDD and rMDD patients showed abnormal HE values in left insula, right cingulate gyrus and left superior temporal gyrus, meaning that these brain regions may be the trait markers for MDD. The insula plays an important role in affective processing, and several studies found that the induced anxiety, retardation and agitation were associated with the insula $[2,44,45]$. The abnormality pattern in the left insula was also consistent with our previous voxelbased morphometry (VBM) study, which reported that the left dorsal anterior insula volume was found atrophy in both cMDD and rMDD groups [42]. The cingulate gyrus is commonly considered as a critical node of default mode network (DMN) [6]. Yao et al. reported a significant correlation between cingulate gyrus and hopelessness, cognitive disturbance and retardation in MDD patients [2], and the cingulate gyrus was inferred to play a role in the modulation of memory by emotionally arousing stimuli [46]. Besides, the superior temporal gyrus exhibited weaker responses to effort-based decision in MDD patients in a recent study [47]. In a word, these regions showed the vulnerability to the MDD, and could be served as the trait markers for MDD.

Compared to rMDD patients, cMDD patients showed significantly abnormal HE value in middle frontal gyrus, inferior parietal lobule, middle occipital gyrus and superior parietal cortex, suggesting that these brain regions may be the state markers for MDD. The middle frontal gyrus and inferior parietal lobule belong to DMN, which supports the self-reflective process, rumination and brooding in depressive patients $[48,49]$. The abnormal fluctuations of both regions could add a complementary evidence for the abnormalities of DMN in MDD patients. The middle occipital gyrus belongs to visual recognition circuit [4], and several previous studies reported aberrant functional activities of middle occipital gyrus in MDD patients $[4,50]$. In addition, an altered superior parietal cortex-caudate correlation pattern was reported in MDD patients in a previous study [51]. All these evidences lead to the conclusion that the depressive state is associated with the functions of these brain regions.

The current study had the following limitations. First, although much information has been acquired through $\mathrm{HE}$ analysis, the HE cannot provide a complete understanding of the neurobiology of depressive disorders. Other measures of complexity are needed for further understanding the mechanisms of MDD in future studies. Second, a relatively small dataset was utilized to estimate the classification accuracy. In the next step, we will collect a larger dataset and incorporate more imaging features to evaluate and improve our method.

\section{MATERIALS AND METHODS}

\section{Subjects}

Participants included 19 cMDD patients, 19 rMDD patients and 19 healthy subjects, and all subjects were females. The cMDD and rMDD patients were confirmed by two expert psychiatrists using the Structured Clinical Interview for DSM-IV (SCID). Totally, the cMDD and rMDD patients were different in their clinical symptoms: the cMDD patients showed depressive state at present while the rMDD patients displayed remitted mental health currently but had past histories as MDD. All depressive patients were rated on 17-item HAMD on the day of scanning. The rMDD patients were defined with HAMD scored no more than 7, while the cMDD patients scored no less than $17[42,52]$. Inclusion criteria for the MDD patients were as follows: (1) meeting the SCID for MDD; (2) between 18 and 65 years old; (3) no history or complication of other psychiatric disorders; (4) able to give voluntary informed consent. Among the participants, one rMDD patients and three cMDD patients were medication free, and other patients were on medication including sodiumvalproate, sertraline, citalopram, lithium and divalproex. Nineteen education level and age well matched healthy subjects were recruited from the local community by print advertisements, and screened with the Non-patient Version Structured Interview from the DSM-IV. The exclusion criteria applied to all subjects includes: contraindications for MRI; with histories of stroke, neurological disorders, major physical diseases, alcohol or drug abuse, and system diseases such as thyroid dysfunction, severe anemia, syphilis or acquired immune deficiency syndrome.

After a complete description of the study to all participants, written informed consent from all subjects was obtained, and this study was approved by Research Ethics Review Board of Beijing Anding Hospital, Capital Medical University and Beijing Normal University Imaging Center for Brain Research. The procedures were carried out in accordance with the approved guidelines.

\section{Image acquisition}

All images were collected on a Siemens $3.0 \mathrm{~T}$ 8 channel MRI scanner. All subjects were instructed to keep their eyes open, relaxed and awake during the scanning. To minimize head motion and instrumental noise effect, a birdcage coil fitted with foam padding was used. Resting-state fMRI data were acquired by gradient echo-planar imaging (EPI) sequence with the following parameters: repetition time $(\mathrm{TR})=2000 \mathrm{~ms}$, echo time $(\mathrm{TE})=30 \mathrm{~ms}$, flip angle $(\mathrm{FA})=90^{\circ}$, slice thickness $=4$ 
$\mathrm{mm}$, slices number $=33$, field of view $(\mathrm{FOV})=220 \times$ $220 \mathrm{~mm}^{2}$, matrix size $=64 \times 64$, and the scan lasted for 8 minutes and 240 volumes were acquired. The structural T1-weighted images were acquired without gap, and TR $=2530 \mathrm{~ms}, \mathrm{TE}=3.39 \mathrm{~ms}, \mathrm{FA}=7^{\circ}$, slice thickness $=1.33$ $\mathrm{mm}, \mathrm{FOV}=256 \times 256 \mathrm{~mm}^{2}$, and voxel dimension $=1 \times$ $1 \times 1.33 \mathrm{~mm}^{3}$.

\section{Data preprocessing}

Data preprocessing were performed via the statistical parametric mapping (SPM8) (http://www. fil. ion.ucl.ac.uk/spm) and Data Processing Assistant for Resting State fMRI (DPARSF) (http://www.restfmri.net/ forum/DPARSF). The first 10 images were discarded in order to make the subjects adapted to the environment and the scanner to be stabilized. At first, the remaining 230 volumes were corrected for the different acquisition time between slices. Then, all images were realigned to the first image to correct inter-scan head motions, and all subjects were included with the displacements less than $2 \mathrm{~mm}$ in the $\mathrm{x}, \mathrm{y}, \mathrm{z}$ axis or the angular motion less than $2^{\circ}$. The spurious covariates including the signals from the ventricular system, white matter and the six head motion parameters obtained from the rigid-body transform were regressed. Then, a temporal band-pass filter $(0.01-0.10 \mathrm{~Hz})$ was applied to the time series to reduce the influences of respiratory and cardiac noise and the linear drift. Next, all resulting images were normalized to the Montreal Neurological Institute (MNI) template, and every voxel was re-sampled to $3 \times 3 \times 3 \mathrm{~mm}^{3}$. At last, all images were smoothed using a $4 \mathrm{~mm}$ full width at half maximum (FWHM) Gaussian kernel.

\section{Estimation of HE}

Rescaled Range analysis, i.e. R/S analysis, can effectively detect the temporal complexity of a time series. The detailed principle of $\mathrm{R} / \mathrm{S}$ analysis is listed as follows: given a time series $X$ and its length is $M$, the time

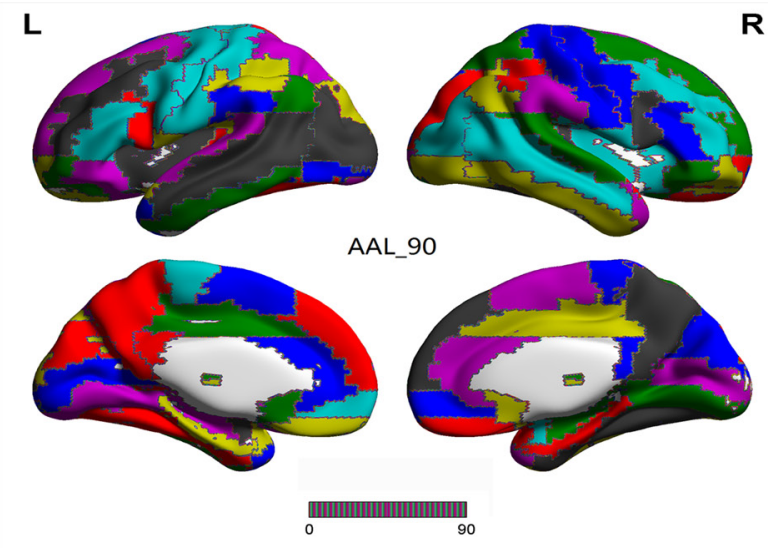

series is divided into $A$ intervals and the length of each interval is $N(1 \leq N \leq A), A \times N=M$. The a-th interval is marked with $I_{a}$ and the k-th element in $I_{a}$ is marked with $\mathrm{x}_{\mathrm{a}, \mathrm{k}}, k=1,2,3 \ldots N$, and $e_{a}$ is the average value in $I_{a}$ interval, then

$$
\begin{aligned}
& y_{a, k}=\sum_{i=1}^{k}\left(x_{a, i}-e_{a}\right) \quad k=1,2 \ldots . . N \\
& R_{a}=\max _{1 \leq k \leq N}\left\{y_{a, k}\right\}-\min _{1 \leq k \leq N}\left\{y_{a, k}\right\} \\
& S_{a}=\left[\frac{1}{N} \sum_{i=1}^{N}\left(x_{a, i}-e_{a}\right)^{2}\right]^{\frac{1}{2}} \\
& (R / S)_{N}=\frac{1}{A} \sum_{a=1}^{N}\left(R_{a} / S_{a}\right)=c N^{H}
\end{aligned}
$$

Where $c$ is a constant, and HE was defined as the slope of the line that fits the pairs $\left(\ln N, \ln (R / S)_{N}\right)$ in a least-square sense.

\section{Feature selection}

The individual HE maps were partitioned into 1024 brain regions and 90 brain regions according to AAL-1024 and AAL-90 atlases (Figure 6). After that, the mean $\mathrm{HE}$ values in each brain region corresponding to two kinds of AAL atlases were served as the candidate features, respectively. Given that some features are redundant and irrelevant for classification, selecting out the discriminative features will improve the final classification performance $[53,54]$. Therefore, two-sample two-tailed t tests were performed on the mean $\mathrm{HE}$ values of every brain region in two AAL atlases to determine the significant between-group differences as the classification features, and the significance level was set at $\mathrm{P}<0.05$. It is worth noting that the feature selection was only performed on the training set of every LOOCV fold, which could reduce the overfitting of the classifier.

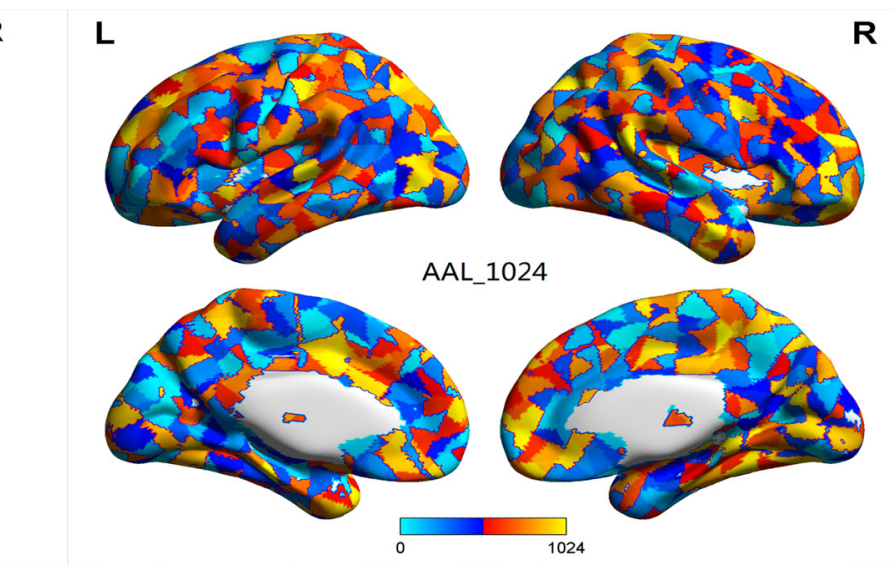

Figure 6: AAL-90 atlas and AAL-1024 atlas 


\section{SVM based method}

In this paper, a SVM based method was proposed to discriminate every pair of the cMDD patients, rMDD patients and HC groups. The SVM algorithms were proposed to find the hyperplane with maximum margin in the feature space. The minimal distance from the closest training samples to the separating hyperplane is called margin, and a larger margin corresponds to a better generalization. The training examples that lie on the margin are called support vectors, which determine the location of hyperplane and could be regarded as the most difficult data to classify. In this paper, LibSVM toolbox (http://www.csie.ntu.edu.tw/ cjlin/libsvm) was used for SVM implementation.

The proposed method adopted a radial basis function (RBF) which is defined as $\left(X, X_{i}\right) \rightarrow K\left(X, X_{i}\right)=e^{\gamma\left|X-X_{i}\right|^{2}}$ as the kernel function, and a grid search method to optimize two parameters: $\gamma$, the width of the RBF, and $C$, an input parameter for the SVM, which adjusts the tradeoff between having zero training errors and allowing misclassifications. The grid search method is referred to as the classification performed with $(\gamma, C)$ varying along a grid with $\gamma=2^{8}, 2^{-7.8}, 2^{-7.5}, \ldots, 2^{8}$ and $C=2^{8}, 2^{-7.3}, 2^{-7.5}, \ldots, 2^{8}$ . The input form for SVM is $\langle x, y\rangle$ where $x$ represents classification features and $y$ stands for the class labels. Here, $x$ represents the mean HE values in the brain regions that showed significant differences between groups, and $y$ is the predefined group label ( 1 or -1$)$ in discriminating every pair of three groups. Classification performance was quantified with accuracy, sensitivity and specificity using LOOCV, and the pair $(\gamma, C)$ with the highest accuracy rate on the $65 \times 65$ grid will be selected as the optimal parameters.

\section{Permutation test}

Permutation test was used to estimate whether the observed classification accuracy was statistically significant. All class labels were randomly permuted to form a new training set, and then the feature selection and the proposed classification method were performed on the new training set. The permutation test was repeated 1000 times, and the 1000 classification accuracies formed the permutation distribution. The $P$ value was calculated as the proportion of the classification accuracy with the randomized label no less than the accuracy with the original label. If $P$ value was less than 0.05 , it demonstrated that the actual accuracy was statistically significant.

\section{Abbreviations}

MDD: major depressive disorder; rMDD: remitted major depressive disorder; cMDD: current major depressive disorder; HC: healthy controls; MRI: Magnetic resonance imaging; ReHo: regional homogeneity; ALFF: amplitude of low-frequency fluctuation; fALFF: fractional amplitude of low-frequency fluctuation; fMRI: functional Magnetic resonance imaging; HE: Hurst exponent; SVM: support vector machine; AAL: Automated Anatomical Labeling; BOLD: blood oxygen level-dependent. RBF: radial basis function; LOOCV: leave-one-out cross-validation; HAMD: Hamilton Depression Rating Scale; ROC: receiver operating characteristics; AUCs: area under the curves; VBM: voxelbased morphometry; DMN: default mode network; SCID: Structured Clinical Interview for DSM-IV; EPI: echo-planar imaging; TR: repetition time; TE: echo time; FA: flip angle; FOV: field of view; SPM: statistical parametric mapping; DPARSF: Data Processing Assistant for Resting State fMRI; MNI: Montreal Neurological Institute; FWMH: full width at half maximum.

\section{Author contributions}

Bin Jing, Zhuqing Long and Han Liu made substantial contributions to the conception, design, analysis and interpretation of data, and drafted the manuscript. Bin Jing and Huagang Yan made contributions to the revision of the manuscript. Jianxin Dong, Xiao Mo and Dan Li made contributions to the conception, design and revision of figures. Chunhong Liu made contributions to the data acquisition. Haiyun $\mathrm{Li}$, the corresponding author, made contributions to conception and interpretation of data, and determined the final version to be submitted for publishing. All authors read and approved the final manuscript.

\section{CONFLICTS OF INTEREST}

There is no conflicts of interest.

\section{FUNDING}

This work was supported by Beijing Natural Science Foundation (No.7174282, 4122018), Medicine and Clinical Cooperative Research Program of Capital Medical University, Grant (No. 13JL04, No. 14JL80, No. 15JL18, No.15JL58, No. 16JL25), National Natural Science Foundation of China, Grant (No. 81471389) and the High level health technical personnel in Beijing, Grant (No. 2014-3-095), and the open project of Laboratory of Brain Disorders, Ministry of Science and Technology (No. 2015NZDJ04).

\section{REFERENCES}

1. Fu CH, Mourao-Miranda J, Costafreda SG, Khanna A, Marquand AF, Williams SC, Brammer MJ. Pattern classification of sad facial processing: toward the development of neurobiological markers in depression. Biol Psychiat. 2008; 63:656-662.

2. Yao Z, Wang L, Lu Q, Liu H, Teng G. Regional homogeneity in depression and its relationship with 
separate depressive symptom clusters: A resting-state fMRI study. J Affect Disorders. 2009; 115:430-438.

3. Wang L, Hermens DF, Hickie IB, Lagopoulos J. A systematic review of resting-state functional-MRI studies in major depression. J Affect Disorders. 2012; 142:6-12.

4. Guo WB, Liu F, Xue ZM, Xu XJ, Wu RR, Ma CQ, Wooderson SC, Tan CL, Sun XL, Chen JD, Liu ZN, Xiao CQ, Chen HF, et al. Alterations of the amplitude of lowfrequency fluctuations in treatment-resistant and treatmentresponse depression: a resting-state fMRI study. Prog Neuro-Psychoph. 2012; 37:153-60.

5. Liu CH, Ma X, Song LP, Tang LR, Jing B, Zhang Y, Li F, Zhou Z, Fan J, Wang CY. Alteration of spontaneous neuronal activity within the salience network in partially remitted depression. Brain Res. 2015; 1599:93-102.

6. Zhang YF, Han Y, Wang YZ, Zhang YF, Jia HX, Jin EH, Deng LG, Li L. Characterization of resting-state fMRIderived functional connectivity in patients with deficiency versus excess patterns of major depression. Complement Ther Med. 2015; 23:7-13.

7. Du MY, Wu QZ, Yue Q, Li J, Liao Y, Kuang WH, Huang XQ, Chan RC, Mechelli A, Gong QY. Voxelwise meta-analysis of gray matter reduction in major depressive disorder. Prog Neuro-Psychoph. 2012; 3:11-6.

8. Gong Q, Wu Q, Scarpazza C, Lui S, Jia Z, Marquand A, Huang X, McGuire P, Mechelli A. Prognostic prediction of therapeutic response in depression using high-field MR imaging. Neuroimage. 2011; 55:1497-503.

9. Strakowski SM, Adler CM, Almeida J, Altshuler LL, Blumberg HP, Chang KD, DelBello MP, Frangou S, McIntosh A, Phillips ML, Sussman JE, Townsend JD. The functional neuroanatomy of bipolar disorder: a consensus model. Bipolar Disord. 2012; 14:313-25.

10. Yuan Y, Zhang Z, Bai F, Yu H, Shi Y, Qian Y, Liu W, You J, Zhang X, Liu Z. Abnormal neural activity in the patients with remitted geriatric depression: A resting-state functional magnetic resonance imaging study. J Affect Disorders. 2008; 111:145-52.

11. Jing B, Liu CH, Ma X, Yan HG, Zhuo ZZ, Zhang Y, Wang SH, Li HY, Wang CY. Difference in amplitude of low-frequency fluctuation between currently depressed and remitted females with major depressive disorder. Brain Res. 2013; 1540:74-83.

12. Hahn T, Marquand AF, Ehlis AC, Dresler T, KittelSchneider S, Jarczok TA, Lesch KP, Jakob PM, MouraoMiranda J, Brammer MJ, Fallgatter AJ. Integrating neurobiological markers of depression. Arch Gen Psychiat. 2011; 68:361-8.

13. Wei M, Qin J, Yan R, Li H, Yao Z, Lu Q. Identifying major depressive disorder using Hurst exponent of resting-state brain networks. Psychiat Res-Neuroim. 2013; 214:306-312.

14. Wei M, Qin J, Yan R, Bi K, Liu C, Yao Z, Lu Q. Association of resting-state network dysfunction with their dynamics of inter-network interactions in depression. J Affect Disorders. 2014; 174:527-534.
15. Marquand AF, Mourão-Miranda J, Brammer MJ, Cleare AJ, $\mathrm{Fu} \mathrm{CH}$. Neuroanatomy of verbal working memory as a diagnostic biomarker for depression. Neuroreport. 2008; 19:1507-11.

16. Costafreda SG, Chu C, Ashburner J, Fu CH. Prognostic and Diagnostic Potential of the Structural Neuroanatomy of Depression. Plos One. 2009; 4:e6353.

17. Craddock RC, James GA, Holtzheimer PE, Hu XP, Mayberg HS. A whole brain fMRI atlas generated via spatially constrained spectral clustering. Hum Brain Mapp. 2012; 33:1914-28.

18. Joliot M, Jobard G, Naveau M, Delcroix N, Petit L, Zago L, Crivello F, Mellet E, Mazoyer B, Tzourio-Mazoyer N. AICHA: An atlas of intrinsic connectivity of homotopic areas. J Neurosci Meth. 2015; 254:46-59.

19. Fan L, Li H, Zhuo J, Zhang Y, Wang J, Chen L, Yang Z, Chu C, Xie S, Laird AR, Fox PT, Eickhoff SB, Yu C, et al. The Human Brainnetcome Atlas: A New Brain Atlas Based on Connectional Architecture. Cereb Cortex. 2016; 26: 3508-26.

20. Glasser MF, Coalson TS, Robinson EC, Hacker CD, Harwell J, Yacoub E, Ugurbil K, Andersson J, Beckmann CF, Jenkinson M, Smith SM, Van Essen DC. A multi-modal parcellation of human cerebral cortex. Nature. 2016; 536: 171-8.

21. Fornito A, Zalesky A, Bullmore ET. Network Scaling Effects in Graph Analytic Studies of Human Resting-State fMRI Data. Front Syst Neurosci. 2010; 4:22.

22. Zalesky A, Fornito A, Harding IH, Cocchi L, Yücel M, Pantelis C, Bullmore ET. Whole-brain anatomical networks: Does the choice of nodes matter? Neuroimage. 2010; 50:970-83.

23. Tzourio-Mazoyer N, Landeau B, Papathanassiou D, Crivello F, Etard O, Delcroix N, Mazoyer B, Joliot M. Automated anatomical labeling of activations in SPM using a macroscopic anatomical parcellation of the MNI MRI single-subject brain. Neuroimage. 2002; 15:273-89.

24. Dai Z, Yan C, Wang Z, Wang J, Xia M, Li K, He Y. Discriminative analysis of early Alzheimer's disease using multi-modal imaging and multi-level characterization with multi-classifier (M3). Neuroimage. 2012; 59:2187-95.

25. Wee CY, Yap PT, Zhang D, Denny K, Browndyke JN, Potter GG, Welsh-Bohmer KA, Wang L, Shen D. Identification of MCI individuals using structural and functional connectivity networks. Neuroimage. 2012; 59:2045-56.

26. Zeng LL, Shen H, Liu L, Wang L, Li B, Fang P, Zhou Z, Li Y, Hu D. Identifying major depression using whole-brain functional connectivity: a multivariate pattern analysis. Brain. 2012; 135:1497-507.

27. Ota K, Oishi N, Ito K, Fukuyama H. A comparison of three brain atlases for MCI prediction. J Neurosci Meth. 2014; 221:139-150.

28. Lilia M, Benoit M, Olivier C, Marie S, Valérie HB, Bruno D, Patrick G, Stéphane L, Serge K. Identification of Atrophy Patterns in Alzheimer's Disease Based on SVM Feature 
Selection and Anatomical Parcellation. LNCS. 2008; 5128:124-132.

29. Ciuciu P, Varoquaux G, Abry P, Sadaghiani S, KleinschmidtA. Scale-Free and Multifractal Time Dynamics of fMRI Signals during Rest and Task. Front Physiol. 2012; 3:186.

30. Maxim V, Sendur L, Fadili J, Suckling J, Gould R, Howard R, Bullmore E. Fractional Gaussian noise, functional MRI, Alzheimer's disease. Neuroimage. 2005; 25:141-58.

31. Park C, Lazar NA, Ahn J, Sornborger A. A multiscale analysis of the temporal characteristics of resting-state fMRI data. J Neurosci Meth. 2010; 193:334-342.

32. Gentili C, Vanello N, Cristea I, David D, Ricciardi E, Pietrini P. Proneness to social anxiety modulates neural complexity in the absence of exposure: A resting state fMRI study using Hurst exponent. Psychiat Res-Neuroim. 2015; 232:135-44.

33. Wink AM, Bernard F, Salvador R, Bullmore E, Suckling J. Age and cholinergic effects on hemodynamics and functional coherence of human hippocampus. Neurobiol Aging. 2006; 27:1395-404.

34. Lai MC, Lombardo MV, Chakrabarti B, Sadek SA, Pasco G, Wheelwright SJ, Bullmore ET, Baron-Cohen S; MRC AIMS Consortium, Suckling J. A shift to randomness of brain oscillations in people with autism. Biol Psychiat. 2010; 68:1092-9.

35. Hahn T, Dresler T, Ehlis AC, Pyka M, Dieler AC, Saathoff C, Jakob PM, Lesch KP, Fallgatter AJ. Randomness of restingstate brain oscillations encodes Gray's personality trait. Neuroimage. 2012; 59:1842-5.

36. Lei X, Zhao Z, Chen H. Extraversion is encoded by scalefree dynamics of default mode network. Neuroimage. 2013; 74:52-7.

37. Hsu CW, Chang CC, Lin CJ. A practical guide to support vector classification. Available at: https://www.csie.ntu. edu.tw/ cjlin/papers/guide/guide.pdf. 2003.

38. De MF, Valente G, Staeren N, Ashburner J, Goebel R, Formisano E. Combining multivariate voxel selection and support vector machines for mapping and classification of fMRI spatial patterns. Neuroimage. 2008; 43:44-58.

39. Pereira F, Mitchell T, Botvinick M. Machine learning classifiers and fMRI: a tutorial overview. Neuroimage. 2009; 45:S199-209.

40. Sokunbi MO, Gradin VB, Waiter GD, Cameron GG, Ahearn TS,Murray AD, Steele DJ, Staff RT. Nonlinear Complexity Analysis of Brain fMRI Signals in Schizophrenia. Plos One. 2014; 9:e95146.

41. Sheline YI, Barch DM, Price JL, Rundle MM, Vaishnavi SN, Snyder AZ, Mintun MA, Wang S, Coalson RS, Raichle ME. The default mode network and self-referential processes in depression. P Natl Acad Sci USA. 2009; 106:1942-7.

42. Liu CH, Jing B, Ma X, Xu PF, Zhang Y, Li F, Wang YP, Tang LR, Wang YJ, Li HY, Wang CY. Voxel-based morphometry study of the insular cortex in female patients with current and remitted depression. Neuroscience. 2014; 262:190-9.

43. Khazaee A, Ebrahimzadeh A, Babajani-Feremi A. Application of advanced machine learning methods on resting-state fMRI network for identification of mild cognitive impairment and Alzheimer>s disease. Brain Imaging Behav. 2015; 10:799-817.

44. Reiman EM, Lane RD, Ahern GL, Schwartz GE, Davidson RJ, Friston KJ, Yun LS, Chen K. Neuroanatomical correlates of externally and internally generated human emotion. Am J Psychiat. 1997; 154:918-925.

45. Graff-Guerrero A, González-Olvera J, Mendoza-Espinosa Y, Vaugier V, García-Reyna JC. Correlation between cerebral blood flow and items of the Hamilton Rating Scale for Depression in antidepressant-naive patients. J Affect Disorders. 2004; 80:55-63.

46. Maddock RJ. The retrosplenial cortex and emotion: new insights from functional neuroimaging of the human brain. Trends Neurosci. 1999; 22:310-6.

47. Yang XH, Huang J, Lan Y, Zhu CY, Liu XQ, Wang YF, Cheung EF, Xie GR, Chan RC. Diminished caudate and superior temporal gyrus responses to effort-based decision making in patients with first-episode major depressive disorder. Prog Neuro-Psychoph. 2016; 64:52-9.

48. Yao Z, Wang L, Qing LU, Liu H. Altered default mode network functional connectivity in patients with depressive disorders: resting-state fMRI study. Chin J Nervous Mental Dis. 2008; 34:278-282.

49. Greicius MD, Kiviniemi V, Tervonen O, Vainionpää V, Alahuhta S, Reiss AL, Menon V. Persistent default-mode network connectivity during light sedation. Hum Brain Mapp. 2008; 29:839-47.

50. Cerullo MA, Eliassen JC, Smith CT, Fleck DE, Nelson EB, Strawn JR, Lamy M, DelBello MP, Adler CM, Strakowski SM. Bipolar I disorder and major depressive disorder show similar brain activation during depression. Bipolar Disord. 2014; 16:703-12.

51. Yang XH, Wang Y, Huang J, Zhu CY, Liu XQ, Cheung EF, Xie GR, Chan RC. Increased prefrontal and parietal cortical thickness does not correlate with anhedonia in patients with untreated first-episode major depressive disorders. Psychiat Res-Neuroim. 2015; 234:144-51.

52. Li B, Liu L, Friston KJ, Shen H, Wang L, Zeng LL, Hu D. A Treatment-Resistant Default Mode Subnetwork in Major Depression. Biol Psychiat. 2013; 74:48-54.

53. Dosenbach NU, Nardos B, Cohen AL, Fair DA, Power JD, Church JA, Nelson SM, Wig GS, Vogel AC, LessovSchlaggar CN, Barnes KA, Dubis JW, Feczko E, et al. Prediction of individual brain maturity using fMRI. Science. 2010; 329:1358-61.

54. Guyon I, Elisseeff A. An Introduction to Variable Feature Selection. J Mach Learn Res. 2003; 3:1157-1182. 